\title{
Survey on Multiframe Super-Resolution
}

\author{
Jagruti Rajput \\ PG Student \\ Parul Institute of Technology
}

\author{
Bhailal Limbasiya \\ Assistant Professor \\ Parul Institute of Technology
}

\begin{abstract}
Super-resolution technique is used for resolution enhancement. In multiframe super-resolution multiple low resolution images are combined to get high resolution image.This paper addresses multiframe super-resolution in which one or more low resolution images are combined to get high resolution image which can increase spatial resolution of image and different image quality matrices to measure the quality of the original image and reconstructed image.
\end{abstract}

\section{Keywords}

Spatial resolution, multiframe,super-resolution

\section{INTRODUCTION}

Image Super-Resolution ${ }^{[1]}$ is most widely used research area and it can solve the limited resolution problem by image acquisition devices and sensor. But, high resolution sensor is very expensive. So,we can increase the resolution by two ways either by reducing pixel size or by increasing chip size. But it can generate noise and result in degradable image quality. So, there is another method to increase resolution of image. Super-resolution can be used in medical imaging, satellite imaging, remote imaging, enlarging consumer photograph for higher quality etc. Super-resolution techniques can be classified into two major parts: Frequency domain and spatial domain approach. Frequency domain approach can perform fourier transform of an image. These methods are simple and cheap. Spatial domain approch can perform directly on pixel. This method is popular but expensive. Technical implementation of super-resolution can be done in two ways: single-frame and multi-frame. Single-frame superresolution methods generate high-resolution image from single degraded noisy image. Multi-frame super-resolution is to generate high-resolution image from multiple lowresolution images of same scene and increase spatial resolution by fusing information. Multiframe super-resolution can be performed in three steps: Image registration, Image fusion and image interpolation. This paper is organized as follows. In Section II, we describe multiframe superresolution images. In Section II, we describe multiframe super-resolution images. Section III presents multiframe super-resolution techniques. Section IV represents different image quality matrices.

\section{MULTIFRAME SUPER-RESOLUTION IMAGES}

In Multi-frame SR imaging ${ }^{[2]}$, performing the observed LR image from SR image is modeled by:

$$
\mathrm{y}=\mathrm{FHD}_{\mathrm{x}}+\mathrm{n}
$$

Where, $\mathrm{x}$ is an original HR image, $\mathrm{F}$ is linear transformation, $\mathrm{H}$ is a image warping, $\mathrm{D}$ is the down-sampling operator and $\mathrm{n}$ is a noise. In this paper, multi-frame Super-Resolution is to generate the high-resolution (HR) image from multiple lowresolution images perspectives of a same scene and also increase spatial resolution by fusing information. There are three steps for multi-frame SR, 1) Image Registration, which is performed first for alignment of the LR images as accurately as possible, and also estimate movement parameters, 2) Image Fusion, which is the process of combing information of interest in two or more LR images into a single high-resolution image, 3) Interpolation, which is done to get high- resolved image. So, Image registration plays accurate role in image reconstruction process.

\section{MULTIFRAME SUPER-RESOLUTION METHODS}

\subsection{Enhanced Shift And Add Method}

In Enhanced Shift and Add method ${ }^{[3]}$ the first step is to perform nonzero initialization. Nonzero initialization is performed by registering the upsampled frames. It gives better results then registering the LR images followed by upsampling. The registered images are then fused by median filtering. This step solves the problem of undefined pixels. The second step is selective optimization. In this we have an accurate guess $\mathrm{x}_{0}$ by optimizing its lexicographic form . For this, we have robust bilateral total variation. This method only selects pixels that is part of optimization process.

\subsection{Sparse Representation And Matrix Completion \\ Sparse Representation}

In sparse representation and matrix completion method ${ }^{[4]}$ in sparse representation the signal is represented by $x \in R^{n}$ in an Over-complete dictionary pair $D \in R^{m \times n}$ with $Q$ atoms. The observation of an signal $\mathrm{x}$, represented as y denoted by,

$\mathrm{y}=\mathrm{S}_{\mathrm{X}}=\mathrm{SD} \alpha_{0}$

In which $\propto_{0} \in R^{Q}$ is a sparse vector and $\mathrm{S} \varepsilon \mathrm{R}^{\mathrm{m} \times \mathrm{n}}$ is a matrix used to downsample $X$. Let $X$ denote original HR image and $\mathrm{Y}$ is a degraded LR version of $\mathrm{X}$. In this, $\mathrm{X}$ is partitioned into submatrices, called patches of dimension $\mathrm{n} \times \mathrm{n}$. $\mathrm{Y}$ is partitioned into submatrices into $\mathrm{m} \times \mathrm{m}$ LR patches. The relation between index on patch $x_{i}$ and its position in $X$ is arbitrary. This is same for index on patch $y_{i}$ and its position on $Y$. The image degradation between $\mathrm{X}$ and $\mathrm{Y}$ is modeled by

$\mathrm{y}_{\mathrm{i}}=\mathrm{SHx}_{\mathrm{i}}+\mathrm{n}$

In which $\mathrm{H}$ represents the blurring filter. The LR and HR are represented by

$\mathrm{x}_{\mathrm{i}} \approx \mathrm{D}_{\mathrm{H}} \alpha_{\mathrm{i}}$

$\mathrm{y}_{\mathrm{i}} \approx \mathrm{D}_{\mathrm{L}} \alpha_{\mathrm{i}}$

$\left\|\alpha_{\mathrm{i}}\right\|_{0} \ll \mathrm{Q}$

Then we can train the overcomplete dictionary pair. The dictionary pair $\mathrm{D}_{\mathrm{H}}$ and $\mathrm{D}_{\mathrm{L}}$ are trained using known image patches. Then the feature extraction is done using high pass filtering operations.

\section{Matrix Completion}

The matrix completion is to recover in the low dimensional subspace the truly low-rank matrix A from observation matrix $\mathrm{Z}$ under the assumption $\mathrm{E}$ is being zero, where $\mathrm{E}$ is matrix of perturbations 


\subsection{Super-Resolution based on Inhibition} In Super-resolution based Inhibition method ${ }^{[5]}$, we have to first load the new resolution frames and register them with respect to the first low resolution frames. Then we compute the approximation of high resolution image. Then we apply inhibition principle. In inhibition principle we first choose a reliable criterion to measure the quality of images. Then compare the quality of the low resolution images with its associated rough approximated high resolution image based on the criteria and save quality values cooresponding to each low resolution images. Then we compare the quality of the each low resolution image with its associated rough approximated high resolution image. Then we choose (number_of_the_low_resolution_frames)-(resolution factor) ${ }^{2}$ low resolution frames with least absolute value of criteria as mentioned in criteria and omit them from the set of the low resolution frames. Then register these (resolution factor) ${ }^{2}$ low resolution frames with respect to the first low resolution frame and estimate corresponding motion vectors. Then we apply robust super-resolution algorithm to reconstruct high resolution image based on these new set of low resolution images.

\subsection{Super-Resolution based On Cycle- Spinning}

In Cycle spinning method ${ }^{[6]}$ each LR image is interpolated to the values on a HR grid by an interpolation method. Then each interpolated image is first downsampled and upsampled. Then we choose one estimated HR frame as a reference frame and then estimate intrinsic motion information by optical flow algorithm. Finally the HR frames are wrapped back to the reference frame according to shift information and then averaged to final HR reconstructed frame.

\subsection{Iterative Back Projection}

The Iterative back projection method ${ }^{[7]}$ is one of the multiframe super resolution's restoration method which is common in simple but most powerful simulate and correct approach to restoration. This strategy is easy to understand in application. This strategy needs a prior image to start the process and the strategy uses mean of the registered images. By this way before beginning the iterations, we have the base image to build our outcome. The basic idea in this strategy is to generate a simulation image, generate LR images from the simulation image to compare them with the observed counterparts and using the error between them to generate a better quality simulation. As long as condition is not satisfied the iterations will continue. The condition may be a threshold value, the point where the quality stop increasing, or simply a predefined number of iterations. The aim of the IBP method is to minimize the error between simulated LR images and the observed LR images iteratively.

\section{IMAGE QUALITY MATRICES}

In this paper, the quality of reconstructed image is how similar to the original high-resolution image. So, there is number of measures for image quality likes,1) Mean-squared Error (MSE), 2) Peak Signal-to-noise Ratio (PSNR), and 3) Structural Similarity Index Measure (SSIM).

\subsection{Mean-squared Error (MSE)}

Mean-squared error (MSE) $)^{[8]}$ is simply the squared error between a Super-Reconstructed image and the original high- resolution image. The MSE can be expressed as,

$$
M S E=\frac{1}{m n} \sum_{i=0}^{m-1} \sum_{i=0}^{n-1}[I(i, j)-K(i, j)]^{2}
$$

Where I and $\mathrm{K}$ represent the mxn matrices and also compare the images. They performed for the dimensions ' $i$ ' and ' $j$ ' So, $I(i, j)$ represents the value of pixel $(i, j)$ of original image I and $K(i, j)$ represents the value of pixel $(i, j)$ of reconstructed image.

\subsection{Peak Signal-to-noise Ratio (PSNR)}

The Peak Signal-to-Noise Ratio (PSNR) is defined as a measuring of quality of reconstructed image and also comparing with original image. In that MSE is used for two mxn matrices represents with images I and $\mathrm{K}$ and compare the images. The PSNR can be expressed as,

$$
P S N R=20 \bullet \log _{10} \frac{M A x_{I}^{2}}{\sqrt{M S E}}
$$

Here, $M A X_{I}$ perform the maximum possible pixel value of the image. When the pixels are represented 8 bits per sample, this is 255.The PSNR expressed in decibels.

\subsection{Structural Similarity Index Measure (SSIM)}

The structural similarity (SSIM) index ${ }^{[9]}$ was designed to better way the human visual system (HVS) processes structural information. SSIM measures structure of an image, contrast and compare variance and covariance between the two images. The SSIM can be expressed as:

$$
\operatorname{SSIM}(X, Y)=\frac{\left(2 \mu_{x} \mu_{y}+c_{1}\right)\left(2 \sigma_{x} \sigma_{y}+c_{2}\right)}{\left(\mu_{x}^{2}+\mu_{y}^{2}+c_{1}\right)\left(\sigma_{x}^{2}+\sigma_{y}^{2}+c_{2}\right)}
$$

Where, $\mathrm{x}$ and $\mathrm{y}$ are sub images of $\mathrm{X}$ and $\mathrm{Y} ; \mu \mathrm{x}, \mu \mathrm{y}$ are the average of $\mathrm{x}, \mathrm{y} ; \sigma \mathrm{x}, \sigma \mathrm{y}$ are standard deviations of $\mathrm{x}, \mathrm{y} . \mathrm{C} 1$ is set to $\mathrm{C} 1=(0.01 * 255)^{\wedge} 2$ and $\mathrm{C} 2=(0.01 * 255)^{\wedge} 2$. Conclusions

\section{CONCLUSIONS}

In this paper different super-resolution based methods are reviewed that can enhance HR quality with less blur effect and more details. Image similarity measures were used to measure the efficiency of the algorithms. And lastly different image quality matrices reviewed as measure the original image and reconstructed image.

\section{REFERENCES}

[1] S.C.Park, M.K.Park and M.G.Kang, "Super- resolution image reconstruction- A technical review"- IEEE Signal Processing Mag., vol. 20, pp. 21-36, May 2003.

[2] Xuelong Li,Yanting $\mathrm{Hu}$, Xinbo Gao, et.al, "A multiframe image super-resolution method", IEEE Transactions on Signal Processing 90, pp.405-414, 2010.

[3] Kassem Al Ismaeil;Djamila Aouada;Bruno Mirbach, "Multi-Frame Super-Resolution by Enhanced Shift \& Add", 8th International Symposium on Image and Signal Processing and Analysis ,September 4-6, 2013.

[4] Zhuozheng Wang;J.R. Deller, "Multi-Frame Super Resolution Based On Sparse Representation And Matrix Completion", IEEE Transactions on Signal Processing,2013. 
[5] Hamid Palangi; Shohreh Kasaei, "Fast and robust multiframe superresolution using inhibition principle",Fourth International Conference on Digital Telecommunications, 2009.

[6] Yan Cheng, Xiangzhong Fang, Jun Hou, Songyu Yu, "Multiframe super-resolution reconstruction based on cycle-spinning", IEEE Transactions on Signal Processing,2007.

[7] Bareja, M.N; Modi,C.K, "An effective Iterative Back Projection Based Single Image Super Resolution
Approch",International Conference On Communication Systems and Network Technologies, 2012.

[8] Yusra A.Y. Al-Najjar, Dr. Der Chen Soong, "Comparison of Image Quality Assessment: PSNR, HVS, SSIM, UIQI", International Journal of Scientific \& Engineering Research, Volume 3, Issue 8, August2012.

[9] Z. Wang, A. C. Bovik, H. R. Sheikh and E. P. Simoncelli, "Image quality assessment: From error visibility to structural similarity", IEEE Trans. Image Process., vol. 13, no. 4, pp. 600-612, 2004. 